\title{
Erratum to: Mitochondrial control of cell death induced by hyperosmotic stress
}

\author{
Alfredo Criollo $\cdot$ Lorenzo Galluzzi • \\ M. Chiara Maiuri · Ezgi Tasdemir • \\ Sergio Lavandero · Guido Kroemer
}

Published online: 7 March 2013

(c) Springer Science+Business Media New York 2013

\section{Erratum to: Apoptosis (2007) 12:3-18 \\ DOI 10.1007/s10495-006-0328-x}

Errors were inadvertently included in Fig. $6 \mathrm{~b}$ of the article mentioned above. In particular, the immunoblots depicting the levels of $\mathrm{Bcl}-\mathrm{X}_{\mathrm{L}}$ and $\mathrm{Mcl}-1$, which were originally intended to constitute loading controls for the assessment of Bak and Bax depletion, respectively, were both improperly associated with to Bak- and Bax-related immunoblots, due to pasting errors at figure composition. The amended version of the figure is reported below. Neither quantitative determinations nor the conclusions of this article are altered. The authors apologize for these errors.

The online version of the original article can be found under doi:10.1007/s10495-006-0328-x.

A. Criollo · L. Galluzzi - M. Chiara Maiuri - E. Tasdemir ·

G. Kroemer

Institut Gustave Roussy - 39 rue Camille Desmoulins,

F-94805 Villejuif, France

A. Criollo · L. Galluzzi - M. Chiara Maiuri - E. Tasdemir ·

G. Kroemer $(\square)$

INSERM, Unit "Apoptosis, Cancer and Immunity" - 39 rue

Camille Desmoulins, F-94805 Villejuif, France

e-mail: kroemer@igr.fr
A. Criollo · L. Galluzzi - M. Chiara Maiuri - E. Tasdemir ·
G. Kroemer
Faculté de Médecine, Université Paris-Sud XI - 39 rue
Camille Desmoulins, F-94805 Villejuif, France
A. Criollo $\cdot$ S. Lavandero
Department of Biochemistry and Molecular Biology, Faculty of Chemical and Pharmaceutical Sciences, FONDAP Center for Molecular Studies of the Cell, University of Chile, Olivos 1007, Santiago 8380492, Chile 

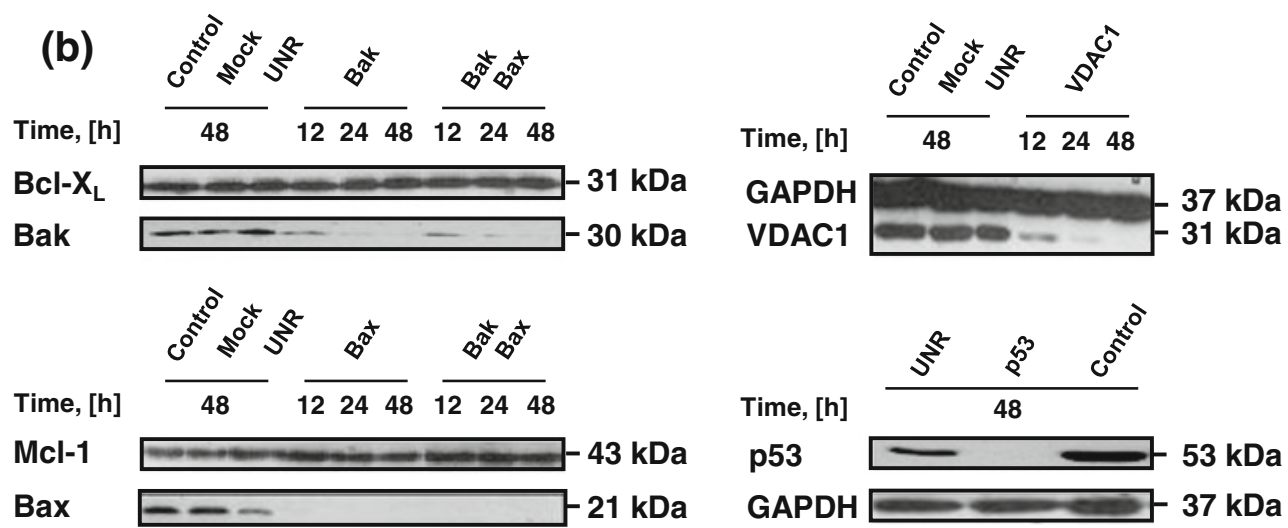

Fig. 6 Involvement of the $B c l-2$ protein family in hyperosmotic stress-induced death of A549 cells. (a) A549 cells were treated with the indicated dose of sorbitol for $6 \mathrm{~h}$, followed by the cytofluorometric assessment of mitochondrial membrane potential ( $\mathrm{DiOC}_{6}(3)$ staining) and viability (PI staining). White columns illustrate the percentage of cells with a low mitochondrial membrane potential but still viable $\left(\mathrm{DiOC}_{6}(3)^{\text {low }}\right)$. Black columns indicate the percentage of cells with disrupted plasma membrane $\left(\mathrm{PI}^{+}\right)$. Data are mean of duplicate experiments \pm SEM. (b, c) A549 cells were transfected with empty liposomes (Mock) or with siRNAs targeting Bax, Bak, Bcl-2, Bcl- $\mathrm{X}_{\mathrm{L}}$, p53, the voltage-dependent anion channel 1 (VDAC1) or an irrelevant "unrelated" control (UNR). (b) To check for the effects of siRNAs, total proteins were purified from transfected A549 cells at different time points $(12,24$ or $48 \mathrm{~h})$, separated according to molecular weight on mono-dimensional SDS-PAGE, and finally analyzed by immunoblotting with the indicated antibodies. Antibodies specific for Mcl-1 and $\mathrm{Bcl}-\mathrm{X}_{\mathrm{L}}$ or for GAPDH were employed as loading controls. (c) A549 cells transfected for $48 \mathrm{~h}$ with the indicated siRNAs were treated with $600 \mathrm{mM}$ sorbitol for additional $6 \mathrm{~h}$, then analyzed at FACS for mitochondrial membrane potential $\left(\mathrm{DiOC}_{6}(3)\right.$ staining) and viability (PI staining). White columns depict the percentage of cells that have dissipated the mitochondrial membrane potential but are still viable $\left(\mathrm{DiOC}_{6}(3)^{\text {low }}\right)$. The percentage of cells with disrupted plasma membrane $\left(\mathrm{PI}^{+}\right)$is illustrated by black columns. Data are mean of duplicate experiments \pm SEM. Dashed lines indicate the range of statistical insignificance from control cells $(p>0.05, \pm 3$ SEM). For additional details please see also "Materials and methods" 